\title{
DSM-5 en pacientes que solicitan el primer tratamiento del trastorno por uso de alcohol. Diferencias de sexo en el estudio multicéntrico CohRTA
}

\section{DSM-5 in patients seeking their first treatment for alcohol use disorder. Sex differences in the multicenter CohRTA study}

\begin{abstract}
Arantza Sanvisens*, Paola Zuluaga*, Gabriel Rubio**, Antoni Short***, Antoni Gual****, Francisco Javier Áldvarez*****, Marta Torrens******, Fernando Rodríguez de Fonseca*******, Roberto MugA*, CohrTA********.

* Servicio de Medicina Interna, Hospital Universitari Germans Trias i Pujol - IGTP, Universitat Autònoma de Barcelona, Badalona. ** Servicio de Psiquiatría, Hospital 12 de Octubre - Instituto i+12, Universidad Complutense de Madrid, Madrid. *** Unidad de Alcohol, Hospital Universitario Son Espases - IdISPa, Palma de Mallorca. **** Servicio de Psiquiatría, Hospital Clínic de Barcelona - IDIBAPS, Universitat de Barcelona, Barcelona. ***** Servicio de Farmacología, Hospital Clínico de Valladolid, Universidad de Valladolid, Valladolid. ****** Instituto de Neuropsiquiatría y Adicciones, Parc de Salut Mar - IMIM, Universitat Autònoma de Barcelona, Barcelona. ******* Instituto de Investigación Biomédica de Málaga - IBIMA, Málaga. $* * * * * * * *$ Ver lista completa de autores.
\end{abstract}

\section{Resumen}

Objetivo: Analizar las diferencias de sexo en los criterios diagnósticos del DSM-5 de los pacientes que solicitan un tratamiento para el trastorno por uso de alcohol (TUA) por primera vez. Métodos: Pacientes incluidos entre enero 2014 y marzo 2016 en el estudio multicéntrico CohRTA de la Red de Trastornos Adictivos. El diagnóstico del TUA se realizó mediante el DSM-5. Además, se recogieron datos sociodemográficos, sobre el consumo de alcohol y otras sustancias, variables clínicas y una analítica general. Resultados: se incluyeron 313 pacientes (74,8\% hombres); la edad al inicio del primer tratamiento fue de 48,8 años (desviación estándar (DE): 9,9 años), la edad al inicio del consumo de alcohol de 15,9 años (DE: 3,3 años) y la de inicio del consumo regular de 25,6 años (DE: 9,6 años). Un $69,3 \%$ de los pacientes eran fumadores y un $61 \%$ tenían antecedentes familiares de TUA. Un 7,7\% eran consumidores de cocaína y un 18,2\% de cannabis. Las mujeres iniciaron el consumo regular de alcohol más tarde que los hombres $(\mathrm{p}<, 001)$ y usaban benzodiacepinas con mayor frecuencia ( $\mathrm{p}=, 013)$. Según el DSM-5, el 89,5\% de los pacientes presentaban un TUA grave ( $\geq 6$ criterios). En el análisis ajustado (regresión logística), los hombres tenían mayor probabilidad de presentar el criterio diagnóstico relacionado con el incumplimiento de los deberes fundamentales en el trabajo o en el hogar $(\mathrm{OR}=1,92$, IC95\%: 1,06-3,48) y el criterio diagnóstico de consumir alcohol en situaciones de riesgo físico (OR=3,00, IC95\%: 1,655,46). Discusión: El DSM-5 detecta diferencias de sexo en pacientes que solicitan el primer tratamiento del TUA. El deterioro social y el consumo de alcohol de riesgo son significativamente más frecuentes en hombres. Palabras clave: Trastorno por uso de alcohol; DSM-5; Diferencias de sexo.

\begin{abstract}
Objective: We aimed to analyze sex differences in the DSM-5 criteria among patients admitted to their first treatment of alcohol use disorder (AUD). Methods: Assessment of AUD was carried out using DSM-5 diagnostic criteria in a multicenter study (CohRTA) within the Spanish Network on Addictive Disorders. Further, baseline questionnaires including socio-demographics, family history, lifetime alcohol consumption and other substance use, as well as clinical and laboratory parameters were obtained during admission. Results: 313 patients $(74.8 \% \mathrm{M})$ were eligible; mean age at first AUD treatment was 48.8 years (standard deviation $(\mathrm{SD}): 9.9$ years). Age at onset of alcohol use was 15.9 years (SD: 3.3 years) and age at starting regular alcohol consumption was 25.6 years (SD: 9.6 years). Almost $69.3 \%$ of patients were tobacco smokers and $61 \%$ had family history of AUD. Regarding other substance use, $7.7 \%$ were current cocaine users and $18.2 \%$ were cannabis users. Women started regular alcohol consumption later than men $(\mathrm{p}<.001)$ and used benzodiazepines more frequently $(p=.013)$. According to DSM-5, 89.5\% of cases had severe AUD $(\geq 6$ criteria). In the adjusted analysis (logistic regression), men were more likely to neglect major rules $(\mathrm{OR}=1.92,95 \% \mathrm{CI}$ : $1.06-3.48)$ and to have hazardous alcohol use (OR=3.00, 95\%CI: 1.65-5.46). Discussion: DSM-5 detects sex differences in patients seeking their first AUD treatment. Social impairment and risky alcohol use are significantly more frequent in men.
\end{abstract}

Key Words: Alcohol use disorder; DSM-5; Sex differences.

Recibido: Abril 2018; Aceptado: Diciembre 2018

Enviar correspondencia a: Dr. Roberto Muga.

Servicio de Medicina Interna. Hospital Universitari Germans Trias i Pujol, Ctra. Canyet s/n, 08916, Badalona.

E-mail: rmuga.germanstrias@gencat.cat. 
$\mathrm{E}$ 1 consumo de alcohol está ampliamente difundido en la sociedad occidental, donde la prevalencia de abuso y dependencia de alcohol es elevada. En Europa occidental, los hombres muestran una mayor prevalencia de abuso de alcohol y de otras sustancias que las mujeres (European Monitoring Centre for Drugs and Drug Addiction, 2015; Observatorio Español de las Drogas y las Toxicomanías, 2011; Teesson et al., 2010). Sin embargo, los datos poblacionales indican que la prevalencia del consumo de alcohol en hombres y mujeres puede ser similar en edades comprendidas entre los 15 y los 35 años (Observatorio Español de las Drogas y las Adicciones, 2017). Esta reducción en la brecha de género sugiere que los problemas relacionados con el consumo excesivo de alcohol pueden ser cada vez más frecuentes en las mujeres.

En los Estados Unidos de América, el consumo de alcohol de riesgo y el trastorno por uso de alcohol (TUA) en las mujeres ha aumentado en un $60 \%$ y $80 \%$, respectivamente, al comparar los datos del periodo 2001-2002 con los del 2012-2013 (Grant et al., 2017). Además, el incremento en las urgencias relacionadas con el abuso de alcohol ha sido mayor en las mujeres que en los hombres (White, Slater, $\mathrm{Ng}$, Hingson y Breslow, 2018). Algunos estudios ponen de manifiesto que las mujeres muestran una menor probabilidad de pedir un tratamiento del TUA que los hombres, desarrollan más rápidamente un síndrome de dependencia al alcohol y tardan más tiempo en recibir el diagnóstico de TUA (Bravo, Gual, Lligoña y Colom, 2013; Johnson, Richter, Kleber, McLellan y Carise, 2005; Rehm, Manthey, Struzzo, Gual y Wojnar, 2015); todo ello pudiera contribuir a un peor pronóstico del trastorno en la mujer.

El efecto perjudicial del alcohol en la salud presenta una considerable variabilidad clínica, que puede ser debida a múltiples factores biopsicosociales; entre los biológicos, destaca la diferente metabolización del etanol en hombres y mujeres (Crabb, Matsumoto, Chang y You, 2004). La actividad de la enzima alcohol deshidrogenasa gástrica es menor en las mujeres y ello implica que a igual cantidad de alcohol ingerido, las mujeres alcanzan mayor concentración de etanol (Lieber, 2000; Mezey, 2000). De hecho, es bien conocido que la mujer presenta mayor vulnerabilidad a la lesión orgánica inducida por el alcohol (Lieber, 2000). Beber alcohol en exceso también puede impactar en los niveles plasmáticos de las hormonas sexuales, que a su vez son importantes mediadoras de la respuesta inmunológica frente a diferentes patógenos (Bouman, Heineman y Faas, 2005; Mezey, 2000). Además, son varias las hipótesis sobre la mayor susceptibilidad genética de las mujeres al consumo de alcohol (Bravo et al., 2013; Wodarz et al., 2003).

En cuanto a los aspectos psicosociales y laborales, se ha descrito que las mujeres son más vulnerables y muestran mayor riesgo de sufrir efectos perjudiciales sobre la salud (Djindjic, Jovanovic, Djindjic, Jovanovic y Jovanovic, 2012; Hallman, Burell, Setterlind, Odén y Lisspers, 2001). El cre- ciente protagonismo social y laboral de las mujeres pudiera haber influido en su incorporación al consumo de alcohol. Además, el estado civil, la maternidad, y/o el consumo de sustancias en la pareja se han asociado con un mayor riesgo de consumo de alcohol en la mujer (Bríñez Horta, 2001). Sin embargo, para otros autores, la convergencia de patrones de consumo de alcohol no se explicaría únicamente por los cambios socio-laborales de la mujer (White et al., 2015).

El DSM-5 es una clasificación diagnóstica de los trastornos mentales establecida por la Asociación Americana de Psiquiatría (American Psychiatric Association, 2013). Para el trastorno por uso de sustancias, esta versión incluye cambios respecto al DSM-IV, como combinar el abuso y la dependencia en un único criterio diagnóstico, añadir el deseo persistente de consumo (craving) como un nuevo criterio y estratificar la gravedad del trastorno en tres categorías (Bartoli, Carrà, Crocamo y Clerici, 2015; Hasin et al., 2013). Según el DSM-5, se diagnostica un TUA si una persona muestra, en los últimos 12 meses, al menos 2 de los 11 criterios sintomáticos que se contemplan; la gravedad del trastorno se determina en función del número de criterios. Determinar la gravedad del TUA es importante porque los pacientes con trastorno moderado o grave pueden beneficiarse de un tratamiento intensivo (Edelman y Fiellin, 2016). Un estudio multinacional utilizando el DSM-5, muestra que la prevalencia del TUA en adultos difiere entre países (Slade et al., 2016). En España, se estima que hasta el $5 \%$ de la población general adulta pudiera cumplir los criterios diagnósticos de un TUA según el Cuestionario de Identificación de los Trastornos debidos al Consumo de Alcohol (Alcohol Use Disorder Identification Test-AUDIT) (Observatorio Español de las Drogas y las Adicciones, 2017). Sin embargo, todavía disponemos de poca información clínica cuando se utiliza el DSM-5 como una herramienta diagnóstica.

El estudio multicéntrico de la Red de Trastornos Adictivos (CohRTA) se centra en pacientes que solicitan un tratamiento del TUA por primera vez. Analizar los casos durante el primer tratamiento del trastorno nos permite conocer sus características clínicas contemporáneas y evita la selección de pacientes crónicos, con múltiples tratamientos previos. El objetivo primario de este estudio fue utilizar el DSM-5 para describir las diferencias de sexo en aquéllos que solicitan un tratamiento del trastorno por primera vez.

\section{Metodología}

Estudio transversal en pacientes que solicitan un primer tratamiento del TUA en centros públicos de atención primaria u hospitalaria. En el estudio participan, a diciembre de 2017, nueve centros (Hospital del Mar, Hospital Clínic de Barcelona, Hospital Universitari de Bellvitge, Hospital Universitari Germans Trias i Pujol, Centro Municipal de Atención a las Drogodependencias de Badalona (Centro Delta), 
Hospital Clínico de Salamanca, Alcohólicos Rehabilitados de Valladolid, Hospital Universitario 12 de Octubre y Hospital Universitari Son Espases), de cuatro comunidades autónomas (Cataluña, Castilla y León, Islas Baleares y Madrid).

El estudio se aprobó por el Comité Ético de Investigación Clínica (CEIC) del centro coordinador (Hospital Universitari Germans Trias i Pujol) y por los respectivos CEIC de los centros participantes. Los pacientes incluidos en CohRTA firmaron el consentimiento informado que incluía la cesión de datos y de muestras biológicas. Los métodos utilizados en este estudio cumplen con las normas éticas para las investigaciones médicas y los principios de buena práctica clínica establecidos en la Declaración de Helsinki.

El cuestionario basal del estudio incluye características sociodemográficas, antecedentes familiares del trastorno, variables relacionadas con el consumo de alcohol (edad de inicio, edad de inicio del consumo regular, cantidad, tiempo total acumulado de abstinencia hasta el inicio del primer tratamiento del trastorno, número de intoxicaciones etílicas que requirieron atención en urgencias) y con el consumo de otras sustancias (consumo en el último mes de tabaco, cannabis, anfetaminas, benzodiacepinas, opiáceos, antecedente de consumo de drogas por vía parenteral), y los parámetros de una analítica general de sangre incluyendo hemograma (hemoglobina), coagulación (tiempo de protrombina) y bioquímica (sodio, potasio, aspartato aminotransferasa [AST], alanina aminotransferasa [ALT], gama glutamil transpeptidasa [GGT], colesterol total, albúmina, bilirrubina total, urea y creatinina). Así mismo, se recogieron muestras de sangre que se almacenan en el biobanco de la Red de Trastornos Adictivos (Universidad Miguel Hernández, Alicante). Más detalles del protocolo de trabajo que sigue el estudio han sido publicados previamente (Sanvisens et al., 2018).

El diagnóstico de TUA mediante el DSM-5 utiliza 11 criterios y evalúa cuatro grandes áreas: 1) El control deficitario sobre el consumo de la sustancia (cantidades superiores a las previstas, duración del consumo más prolongada de lo previsto, intentos fracasados de abandonar el consumo, invertir mucho tiempo en consumir, deseo persistente de consumir), 2) El deterioro social (incumplimiento de los deberes fundamentales en el trabajo o en el hogar, reducción o abandono de las actividades sociales, problemas en la esfera social), 3) El consumo de riesgo (consumir en situaciones de riesgo físico, consumir a pesar de tener problemas físicos/psicológicos derivados del consumo) y, 4) Los criterios farmacológicos (tolerancia a la sustancia y abstinencia).

La gravedad del trastorno se estratifica en tres niveles: 1) leve (2-3 criterios diagnósticos), 2) moderado (4-5 criterios), y 3 ) grave ( $\geq 6$ criterios).

Entre enero de 2014 y marzo de 2016, 369 pacientes cumplieron el criterio de inclusión en el estudio CohRTA. Para este estudio, se excluyeron 56 pacientes por no dispo- ner de todos los criterios del DSM-5. No obstante, la edad al solicitar el primer tratamiento $(\mathrm{p}=, 60)$, el sexo $(\mathrm{p}=, 36)$ y la edad de inicio del consumo de alcohol $(p=, 51)$ resultaron similares entre los 313 pacientes que entraron en el análisis y los 56 pacientes excluidos.

\section{Análisis estadístico}

Se realizó un análisis descriptivo de los datos en el que las variables categóricas se describieron mediante las frecuencias relativas y las variables continuas mediante la media \pm desviación estándar (DE). Se realizaron análisis bivariados para establecer las diferencias entre los hombres y las mujeres utilizando pruebas chi-cuadrado y F-Fisher para la comparación de variables categóricas y t-Student para determinar las diferencias de medias.

El análisis ajustado se realizó mediante modelos de regresión logística utilizando cada criterio diagnóstico del DSM-5 como variable dependiente. Como variables independientes se incluyeron aquéllas que mostraron diferencias estadísticamente significativas entre los hombres y las mujeres en el análisis univariado.

Los valores de $\mathrm{p}<, 05$ se consideraron estadísticamente significativos. El análisis estadístico fue realizado con Stata software (versión 11,0, College Station, Texas, USA).

\section{Resultados}

Un total de 313 pacientes $(74,8 \%$ hombres) fueron incluidos; la media de edad $\pm \mathrm{DE}$ al inicio del primer tratamiento fue de 48,8 \pm 9,9 años, la edad al inicio del consumo de alcohol fue de 15,9 \pm 3,3 años y la del inicio del consumo regular de alcohol de 25,6 \pm 9,6 años; el 69,3\% de los pacientes eran fumadores de tabaco y un $61 \%$ tenían antecedentes familiares de TUA.

Respecto al uso de otras sustancias, el 7,7\% de los pacientes eran consumidores de cocaína y $18,2 \%$ de cannabis.

En la analítica general, los pacientes mostraban una media de hemoglobina de 13,9 $\pm 2,5 \mathrm{~g} / \mathrm{dL}$, una media de colesterol total de $209 \pm 49 \mathrm{mg} / \mathrm{dL}$ y una media de albúmina de $39,8 \pm 8,8 \mathrm{~g} / \mathrm{L}$. Un $46 \%$ presentaba AST $>40 \mathrm{U} / \mathrm{L}$, un $32,8 \%$ ALT $>40$ U $/$ L y $67,2 \%$ GGT $>50$ U $/$ L. Las Tablas 1 y 2 muestran las características socio-demográficas, los resultados de la analítica general y las características del consumo de alcohol y otras sustancias al inicio del tratamiento.

Las mujeres iniciaron el consumo regular de alcohol más tarde (6 años en promedio) $(\mathrm{p}<, 001)$ y presentaron una mayor prevalencia de consumo de benzodiacepinas ( $\mathrm{p}=, 013)$ que los hombres (Tabla 2).

Globalmente, los criterios del DSM-5 más prevalentes fueron: consumir alcohol en cantidades superiores o periodos más prolongados de los previstos $(97,1 \%)$, seguir consumiendo a pesar de sufrir problemas físicos o psicológicos causados o exacerbados por el alcohol $(91,4 \%)$ y presentar signos de tolerancia a la sustancia $(85,9 \%)$. Un 
Tabla 1. Características sociodemográficas y analíticas de 313 pacientes admitidos a tratamiento del TUA.

\begin{tabular}{|c|c|c|c|c|}
\hline & $\begin{array}{c}\text { Total } \\
\mathrm{N}=313 \\
\text { n (\%) }\end{array}$ & $\begin{array}{c}\text { Hombres } \\
\text { N=234 } \\
\text { n (\%) }\end{array}$ & $\begin{array}{c}\text { Mujeres } \\
\mathrm{N}=79 \\
\mathrm{n}(\%)\end{array}$ & p valor \\
\hline Edad, media $\pm \mathrm{DE}$ & $48,8 \pm 9,9$ & $48,6 \pm 10,1$ & $49,1 \pm 9,1$ & ,726 \\
\hline Nacidos en España & $299(95,5)$ & $225(96,1)$ & $74(93,7)$ & ,356 \\
\hline \multicolumn{5}{|l|}{ Nivel de estudios $(n=303)$} \\
\hline No sabe leer ni escribir & $5(1,7)$ & $4(1,8)$ & $1(1,3)$ & \multirow{4}{*}{,710 } \\
\hline Enseñanza primaria & $65(21,4)$ & $51(22,6)$ & $14(18,2)$ & \\
\hline Enseñanza secundaria & $181(59,7)$ & $135(59,7)$ & $46(59,7)$ & \\
\hline Universitarios & $52(17,2)$ & $36(15,9)$ & $16(20,8)$ & \\
\hline \multicolumn{5}{|l|}{ Estado civil $(n=309)$} \\
\hline Soltero/a & $77(25,0)$ & $61(26,3)$ & $16(20,7)$ & \multirow{3}{*}{, 328} \\
\hline Casado/a o pareja de hecho & $132(42,7)$ & $101(43,5)$ & $31(40,3)$ & \\
\hline Viudo/a, separado/a o divorciado/a & $100(32,3)$ & $70(30,2)$ & $30(39,0)$ & \\
\hline \multicolumn{5}{|l|}{ Situación laboral $(n=309)$} \\
\hline Trabajando & $144(46,6)$ & $113(48,6)$ & $31(40,3)$ & \multirow{3}{*}{$<, 001$} \\
\hline Parado/a, incapacidad permanente, pensionista & $155(50,2)$ & $117(50,5)$ & $38(49,3)$ & \\
\hline Estudiantes/Tareas hogar/Otras & $10(3,2)$ & $2(0,9)$ & $8(10,4)$ & \\
\hline \multicolumn{5}{|l|}{ Convivencia $(n=308)$} \\
\hline Vive solo/a & $53(17,2)$ & $40(17,3)$ & $13(16,9)$ & \multirow{4}{*}{,198 } \\
\hline Con pareja y/o hijos & $169(54,9)$ & $120(51,9)$ & $49(63,6)$ & \\
\hline Con familia de origen & $70(22,7)$ & $59(25,6)$ & $11(14,3)$ & \\
\hline Otras situaciones & $16(5,2)$ & $12(5,2)$ & $4(5,2)$ & \\
\hline Analítica general al inicio del tratamiento del TUA & \multicolumn{4}{|c|}{ media $\pm \mathrm{DE}$} \\
\hline Hemoglobina $(\mathrm{g} / \mathrm{dL})(\mathrm{n}=298)$ & $13,9 \pm 2,5$ & $14,5 \pm 2,4$ & $12,7 \pm 2,3$ &, 010 \\
\hline Tiempo de protrombina (INR) $(n=277)$ & $1,0 \pm 0,2$ & $1,0 \pm 0,2$ & $1,0 \pm 0,1$ & ,436 \\
\hline Colesterol total $(\mathrm{mg} / \mathrm{dL})(\mathrm{n}=279)$ & $209 \pm 49$ & $204 \pm 45$ & $219 \pm 56$ & ,255 \\
\hline Albúmina $(\mathrm{g} / \mathrm{L})(\mathrm{n}=279)$ & $39,8 \pm 8,8$ & $41,3 \pm 7,4$ & $36,8 \pm 10,7$ & ,073 \\
\hline Bilirrubina total $(\mathrm{mg} / \mathrm{dL})(\mathrm{n}=279)$ & $0,9 \pm 0,7$ & $0,9 \pm 0,6$ & $0,8 \pm 0,8$ &, 665 \\
\hline Sodio $(\mathrm{mEq} / \mathrm{L})(\mathrm{n}=298)$ & $139 \pm 3,2$ & $139 \pm 3,4$ & $139 \pm 2,6$ &, 607 \\
\hline Potasio $(\mathrm{mEq} / \mathrm{L})(\mathrm{n}=298)$ & $4,4 \pm 0,4$ & $4,4 \pm 0,5$ & $4,4 \pm 0,3$ & ,876 \\
\hline AST (U/L) $(n=279)$ & $59,4 \pm 50,5$ & $61,2 \pm 50,0$ & $55,4 \pm 53,0$ & ,710 \\
\hline $\operatorname{ALT}(U / L)(n=279)$ & $42,6 \pm 31,9$ & $46,3 \pm 33,5$ & $34,5 \pm 27,0$ & ,185 \\
\hline GGT (U/L) $(n=279)$ & $245 \pm 504$ & $305 \pm 594$ & $116 \pm 141$ &, 157 \\
\hline Urea $(m g / d L)(n=298)$ & $22,6 \pm 9,4$ & $24,4 \pm 9,3$ & $18,6 \pm 8,5$ &, 028 \\
\hline Creatinina $(\mathrm{mg} / \mathrm{dL})(\mathrm{n}=298)$ & $0,7 \pm 0,2$ & $0,8 \pm 0,2$ & $0,6 \pm 0,1$ &, 002 \\
\hline
\end{tabular}

$73,5 \%$ de los casos tenían signos o síntomas de abstinencia al alcohol y un $78,3 \%$ mostraron un deseo persistente de consumir la sustancia o craving.

El $89,5 \%$ de los casos presentaban un TUA grave $(\geq 6$ criterios DSM-5), el 9,3\% moderado (4-5 criterios) y el $1,3 \%$ leve (2-3 criterios), sin observarse diferencias de sexo $(\mathrm{p}=, 487)$.

En la Tabla 3 se muestran las diferencias de sexo para los criterios diagnósticos del DSM-5. En concreto, haber presentado criterios de abstinencia $(\mathrm{p}=, 038)$, consumir alcohol en situaciones de riesgo físico $(\mathrm{p}<, 001)$, incumplir los deberes fundamentales en el trabajo o en el hogar $(\mathrm{p}=, 001)$ y abandonar o reducir las actividades sociales, profesionales o de ocio $(\mathrm{p}=, 064)$ fueron criterios diagnósticos más prevalentes en hombres respecto a las mujeres.

La Figura 1 muestra la probabilidad de presentar los criterios diagnósticos del DSM- 5 en los hombres respecto a las mujeres ajustando por la edad de inicio del consumo regular de alcohol, el consumo de benzodiacepinas y la situación laboral. El análisis de regresión logística indica que los hombres mostraron una probabilidad hasta 3 veces mayor que las mujeres de consumir alcohol en situaciones de riesgo físico (Odds Ratio $(\mathrm{OR})=3,00$, Intervalo de Confianza (IC) $95 \%: 1,65-5,46)$, además de una mayor probabilidad de incumplir con los deberes fundamentales $(\mathrm{OR}=1,92$, IC95\%: 1,06-3,48). 
Tabla 2. Características del consumo de alcohol y otras sustancias de 313 pacientes con TUA.

\begin{tabular}{|c|c|c|c|c|}
\hline & $\begin{array}{c}\text { Total } \\
\mathrm{N}=313 \\
\text { n (\%) }\end{array}$ & $\begin{array}{c}\text { Hombres } \\
\mathrm{N}=234 \\
n(\%)\end{array}$ & $\begin{array}{c}\text { Mujeres } \\
\mathrm{N}=79 \\
\mathrm{n}(\%)\end{array}$ & p valor \\
\hline Edad de inicio del consumo de alcohol, media $\pm D E$ & $15,9 \pm 3,3$ & $15,7 \pm 3,1$ & $16,7 \pm 3,8$ &, 016 \\
\hline Edad de inicio del consumo regular de alcohol, media $\pm D E$ & $25,6 \pm 9,6$ & $24,0 \pm 8,6$ & $30,5 \pm 11,0$ & $<, 001$ \\
\hline Consumo de alcohol (g/día), media $\pm D E$ & $134,8 \pm 80,6$ & $138,7 \pm 86,2$ & $123,1 \pm 60,2$ & ,316 \\
\hline Tiempo total acumulado de abstinencia al alcohol (años), $(\mathrm{n}=307)$, media $\pm D E$ & $2,1 \pm 3,4$ & $2,1 \pm 3,4$ & $2,2 \pm 3,7$ & ,843 \\
\hline Antecedentes familiares de TUA $(n=307)$ & $188(61,0)$ & $138(60,3)$ & $50(64,1)$ &, 548 \\
\hline \multicolumn{5}{|l|}{ Número de intoxicaciones etílicas a lo largo de la vida $(n=304)$} \\
\hline Ninguna & $132(43,4)$ & $98(43,4)$ & $34(43,6)$ & \multirow[t]{3}{*}{ 977 } \\
\hline $1-5$ & $159(52,3)$ & $118(52,2)$ & $41(52,6)$ & \\
\hline$>5$ & $13(4,3)$ & $10(4,4)$ & $3(3,8)$ & \\
\hline \multicolumn{5}{|l|}{ Tabaco } \\
\hline No & $62(19,8)$ & $47(20,1)$ & $15(19,0)$ & \multirow{2}{*}{, 137} \\
\hline Exfumador & $34(10,9)$ & $30(12,8)$ & $4(5,1)$ & \\
\hline \multicolumn{5}{|l|}{ Consumo en el último mes: } \\
\hline Cocaína & $24(7,7)$ & $18(7,7)$ & $6(7,6)$ & ,978 \\
\hline Cannabis/marihuana $(n=312)$ & $57(18,2)$ & $47(20,2)$ & $10(12,7)$ &, 135 \\
\hline Anfetaminas & $6(1,9)$ & $5(2,4)$ & $1(1,3)$ &, 625 \\
\hline Benzodiacepinas & $18(5,7)$ & $9(3,8)$ & $9(11,4)$ &, 013 \\
\hline Opiáceos & $1(0,3)$ & $1(0,4)$ & $0(0)$ &, 561 \\
\hline Antecedente de uso de drogas por vía parenteral $(n=311)$ & $9(2,9)$ & $6(2,6)$ & $3(3,8)$ &, 579 \\
\hline
\end{tabular}

Tabla 3. Diferencias de sexo en los criterios diagnósticos y gravedad del TUA según DSM-5 en 313 pacientes admitidos a tratamiento por primera vez.

\begin{tabular}{|c|c|c|c|c|}
\hline & $\begin{array}{c}\text { Total } \\
\text { N=313 } \\
\text { n (\%) }\end{array}$ & $\begin{array}{c}\text { Hombres } \\
\mathrm{N}=234 \\
\mathrm{n}(\%)\end{array}$ & $\begin{array}{c}\text { Mujeres } \\
\mathrm{N}=79 \\
\mathrm{n}(\%)\end{array}$ & p valor \\
\hline \multicolumn{5}{|l|}{ DSM-5 } \\
\hline \multicolumn{5}{|l|}{ Control deficitario } \\
\hline Cantidades superiores/tiempo prolongado & $304(97,1)$ & $229(97,9)$ & $75(94,9)$ & ,178 \\
\hline Cantidades superiores a las previstas & $301(96,2)$ & $227(97,1)$ & $74(93,7)$ &, 182 \\
\hline Más tiempo del previsto & $281(89,8)$ & $211(90,2)$ & $70(88,6)$ & ,692 \\
\hline Intentos fracasados para abandonar/controlar el consumo & $262(83,7)$ & $196(83,8)$ & $66(83,5)$ & ,964 \\
\hline Invertir mucho tiempo consumiendo & $183(58,5)$ & $137(58,6)$ & $46(58,2)$ & ,960 \\
\hline Deseo persistente - Craving & $245(78,3)$ & $181(77,3)$ & $64(88,0)$ & ,495 \\
\hline \multicolumn{5}{|l|}{ Deterioro social } \\
\hline Incumplimiento de deberes fundamentales & $196(62,6)$ & $129(55,1)$ & $27(34,2)$ &, 001 \\
\hline Reducción o abandono de actividades sociales & $143(45,7)$ & $114(48,7)$ & $29(36,7)$ & ,064 \\
\hline \multicolumn{5}{|l|}{ Consumo de riesgo } \\
\hline Riesgo físico & $196(62,6)$ & $165(70,5)$ & $31(39,2)$ & $<, 001$ \\
\hline Problemas físicos/psicológicos & $286(91,4)$ & $213(91,0)$ & $73(92,4)$ & ,706 \\
\hline \multicolumn{5}{|l|}{ Criterios farmacológicos } \\
\hline Abstinencia & $230(73,5)$ & $179(76,5)$ & $51(64,6)$ & ,038 \\
\hline Dos o más síntomas & $222(70,9)$ & $171(73,1)$ & $51(64,6)$ & ,149 \\
\hline Consumo para aliviar síntomas & $166(53,0)$ & $131(56,0)$ & $35(44,3)$ &, 072 \\
\hline Tolerancia a la sustancia & $269(85,9)$ & $204(87,2)$ & $65(82,3)$ & ,279 \\
\hline Necesidad de cantidades mayores & $259(82,7)$ & $195(83,3)$ & $64(81,0)$ & ,637 \\
\hline Efecto reducido & $236(75,4)$ & $182(77,8)$ & $54(68,3)$ &, 093 \\
\hline \multicolumn{5}{|l|}{ Gravedad } \\
\hline Número de criterios, media $\pm \mathrm{DE}$ & $10,1 \pm 2,4$ & $10,4 \pm 2,4$ & $9,3 \pm 2,3$ &, 001 \\
\hline Grave ( $\geq 6$ criterios) & $280(89,4)$ & $211(90,2)$ & $69(87,4)$ & \\
\hline
\end{tabular}




\section{Criterios DSM-5}

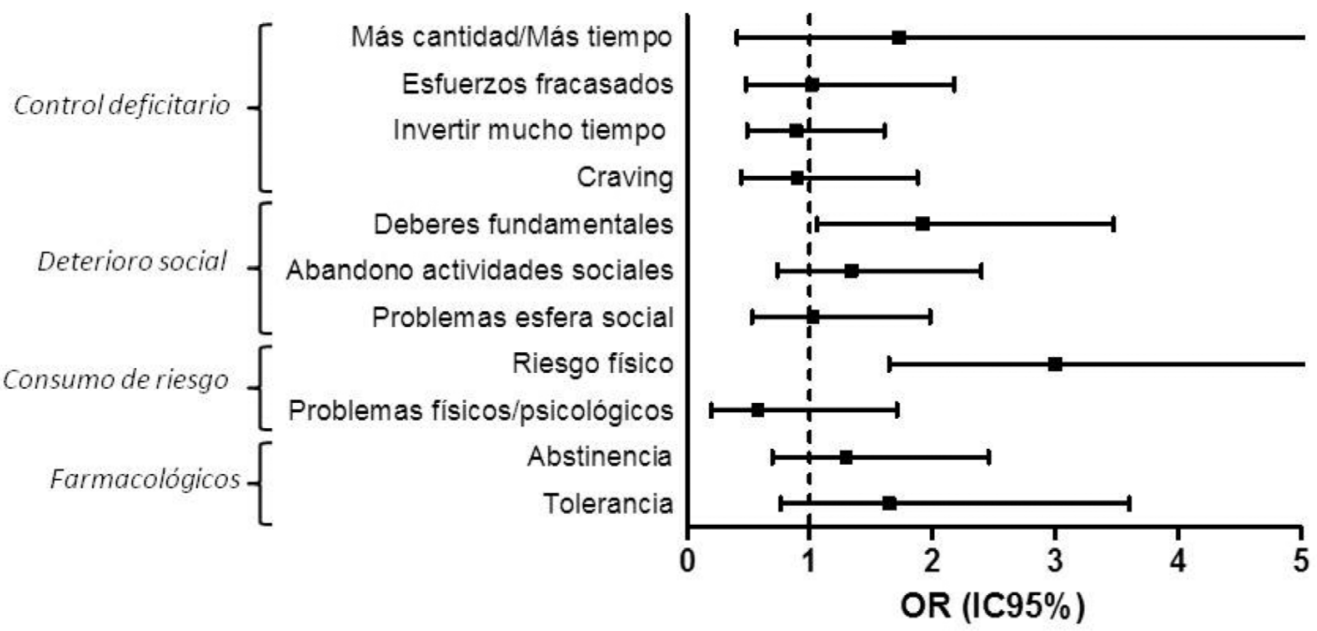

Figura 1. Probabilidad ajustada* (regresión logística) de presentar criterios diagnósticos DSM-5 en hombres respecto a mujeres admitidos a tratamiento de TUA por primera vez.

Nota. * Ajustado por edad de inicio del consumo regular de alcohol, consumo de benzodiacepinas y situación laboral

\section{Discusión}

Este estudio pone de manifiesto que las mujeres con TUA inician el consumo regular de alcohol más tarde que los hombres. Sin embargo, la edad a la que solicitan el primer tratamiento del trastorno resulta similar a la de los hombres sugiriendo que el consumo excesivo de alcohol pudiera evolucionar más rápidamente a TUA en las mujeres.

En este estudio multicéntrico, la edad del primer tratamiento del TUA resulta tardía (casi 50 años para los hombres y las mujeres) y ocurre varias décadas después de iniciar el consumo regular de alcohol, con lo que ello implica en el riesgo de presentar elevada morbilidad cuando solicitan el primer tratamiento. Diversos estudios describen un marcado retraso diagnóstico y terapéutico en el TUA (Chapman, Slade, Hunt y Teesson, 2015; Teesson et al., 2010). Sin embargo, uno reciente en Australia que analiza predictores del retraso, no observa diferencias de sexo (Chapman et al., 2015). Por otro lado, se ha comunicado que las mujeres tienen una menor probabilidad de pedir tratamiento del trastorno, si bien son más cumplidoras del mismo cuando lo inician (Bravo et al., 2013; Rehm et al., 2015). En todo caso, sólo una minoría de las personas con un TUA, solicitaran un tratamiento del trastorno a lo largo de la vida. (Dawson, Goldstein y Grant, 2012; Edlund, Booth y Han, 2012; Rehm et al., 2015).

En el análisis univariado, las mujeres presentan una mayor prevalencia de consumo de benzodiacepinas que los hombres. Este hallazgo coincide con los datos del Observatorio Español sobre Drogas en su encuesta de 2017 sobre el consumo de alcohol y drogas en la población general (Observatorio Español de las Drogas y las Adicciones, 2017). El diseño de este estudio transversal no permite establecer si la mayor prevalencia de consumo de benzodiacepinas por las mujeres es debido a la comorbilidad psiquiátrica u otra sintomatología concurrente como la del síndrome de abstinencia al alcohol que se trata con esta familia de fármacos (Mirijello et al., 2015; Saitz, 2005).

Otra diferencia de sexo observada en el análisis univariado es la situación laboral. Un análisis de las posibles causas sugiere que estas diferencias se deben principalmente a que una parte de las mujeres con un TUA refieren, como actividad laboral, dedicarse a las tareas del hogar (datos no mostrados).

La prevalencia de TUA grave, según el DSM-5, resultó elevada en esta serie de casos que solicitan un tratamiento del trastorno, sin que hubiera diferencias entre los hombres y las mujeres. Sin embargo, el DSM-5 en este estudio detecta diferencias de sexo en determinadas áreas diagnósticas como el deterioro social y el consumo de alcohol en situaciones de riesgo, más probables en los hombres. Estas diferencias pudieran ser debidas a múltiples factores ya sean asociados al estilo de vida u otros; es conocido que los hombres se exponen a situaciones de riesgo con una mayor frecuencia que las mujeres en relación al abuso de alcohol (Schwartz y Davaran, 2013; White, Hingson, Pan y Yi, 2011). Es interesante señalar que cuando se utiliza el DSM-5 para detectar un TUA en la población general, se describen las mismas conclusiones: el consumo recurrente de alcohol en situaciones de riesgo físico y el deterioro social, son siempre más prevalentes en los hombres (Caetano, Gruenewald, Vaeth y Canino, 2018). De hecho, hay una amplia evidencia científica de las diferencias de sexo en algunos procesos neuronales fisiológicos y psicosociales en relación al TUA (Kelly, Ostrowski y Wilson, 1999; Nolen-Hoeksema y Hilt, 2006). 
Por otro lado, algunos factores genéticos puedieran influir en aspectos conductuales del TUA y se dividen en dos grandes grupos: los modificadores genéticos de la neurotransmisión, como los genes que codifican el receptor GABA, y los modificadores genéticos del metabolismo del etanol, en concreto, los polimorfismos de las enzimas implicadas (Anstee, Daly y Day, 2015). En este sentido, sería de interés el disponer de más estudios que muestren la interacción genética y ambiental en el TUA (Salvatore, Cho y Dick, 2017).

Este estudio tiene varias limitaciones que deben ser mencionadas. En primer lugar, al tratarse de un diseño transversal no podemos extraer conclusiones sobre la causalidad de los criterios diagnósticos del DSM-5. Además, en la selección de los pacientes pudiera existir cierto sesgo hacia casos con un TUA grave atendidos en centros públicos, la mayoría de ellos hospitales, respecto a los que son tratados por primera vez en centros de atención primaria, que pudieran ser más jóvenes y con menor comorbilidad. Finalmente, entre las limitaciones del DSM-5 como herramienta diagnóstica se ha argumentado que los criterios diagnósticos son heterogéneos y que su enfoque es excesivamente estricto al analizar la gravedad del trastorno; DSM- 5 no permite distinguir entre abuso y dependencia de alcohol ni determinar la gravedad de cada condición (Helzer, van den Brink y Guth, 2006; Lane y Sher, 2015; Muthén, 2006). Por contra, la fuerza de este estudio multicéntrico se centra en que además del DSM-5 se dispone de otras variables que lo complementan (ej. policonsumo de sustancias), lo que nos permite caracterizar mejor el trastorno. Los resultados aquí presentados revelan la necesidad de adelantar el diagnóstico del TUA y promover su tratamiento en fases más tempranas de la enfermedad.

\section{Agradecimientos}

El Proyecto CohRTA de la RTA está financiado por el Ministerio de Economía y Competitividad, Instituto de Salud Carlos III (RETICS RD16/0017; PI17/174), Fondo Europeo de Desarrollo Regional (FEDER) y por el Plan Nacional Sobre Drogas (PNSD 2014|042, PNSD 2015|027, PNSD2015|054, PNSD 2016|024 y PNSD 2018|020), Ministerio de Sanidad, Política Social e Igualdad.

Paola Zuluaga está adscrita al programa Rio-Hortega, del Instituto de Salud Carlos III (CM17/022).

Esther Papaseit está adscrita al programa Joan Rodés, del Instituto de Salud Carlos III (JR16/00020).

Francina Fonseca y Daniel Fuster están adscritos al Programa d'Intensificació de la Recerca de la Generalitat de Catalunya (SLT006/17/0014 y SLT006/17/0107, respectivamente).

\section{Coautorías del Estudio CohRTA:}

Red de Trastornos Adictivos, Programa Alcohol. Coordinador: Fernando Rodríguez de Fonseca, Instituto de Investigación Biomédica de Málaga -IBIMA, Málaga.

Estudio CohRTA, Comité ejecutivo: Antoni Gual, Marta Torrens, Fernando Rodríguez de Fonseca, Robert Muga.

Proyecto CohRTA, Centro Coordinador: Servicio de Medicina Interna, Hospital Universitari Germans Trias i Pujol, Badalona.

Estudio CohRTA. Centros participantes e investigadores a 1 de Abril de 2018:

Hospital Universitari Germans Trias i Pujol-IGTP, Badalona: Robert Muga, Paola Zuluaga, Daniel Fuster, Marina Simon, Magí Farré, Esther Papaseit, Jordi Tor, Arantza Sanvisens; Hospital Clínic-IDIBAPS, Barcelona: Antoni Gual, Laia Miquel, Lluïsa Ortega, Lidia Teixidor, Chrysanti Blithikioti; Parc de Salut Mar-IMIM, Barcelona: Marta Torrens, Francina Fonseca, Joan Ignasi Mestre-Pinto, María Alías, Claudi Tamarit; Hospital Universitari de Bellvitge-IDIBELL, L'Hospitalet de Llobregat: Ferran Bolao; Hospital Clínico Universitario-IBSAL, Salamanca: Francisco Javier Laso, Miguel Marcos; Hospital 12 de Octubre-Instituto i+12, Madrid: Gabriel Rubio, Ana Sion, Núria García-Marchena; Hospital Clínico Universitario de Valladolid/Alcohólicos Rehabilitados de Valladolid (ARVA), Valladolid: Javier Álvarez, Trinidad Gómez-Talegón; Hospital Universitario Son Espases-IdISPa, Palma de Mallorca: Antoni Short, Catalina Moranta, Rafael Blanes; Centro Municipal de Atención a las Drogodependencias-Centro Delta, Badalona: Inmaculada Rivas, Eva Faure, Néstor Espinach.

Biobanco, Red de Trastornos Adictivos: Jorge Manzanares, Luis Navarro; Instituto de Neurociencias, Universidad Miguel Hernández, San Juan de Alicante.

\section{Conflictos de interés}

No existen.

\section{References}

American Psychiatric Association (2013). Diagnostic and Statistical Manual of Mental Disorders (5th Ed). Washington, DC: American Psychiatric Association.

Anstee, Q., Daly, A. y Day, C. (2015). Genetics of Alcoholic Liver Disease. Seminars in Liver Disease, 35, 361-374. doi:10.1055/s-0035-1567832.

Bartoli, F., Carrà, G., Crocamo, C. y Clerici, M. (2015). From DSM-IV to DSM-5 alcohol use disorder: An overview of epidemiological data. Addictive Behaviors, 41, 46-50. doi:10.1016/j.addbeh.2014.09.029.

Bouman, A., Heineman, M. J. y Faas, M. M. (2005). Sex hormones and the immune response in humans. $\mathrm{Hu}$ man Reproduction Update, 11, 411-423. doi:10.1093/humupd/dmi008. 
Bravo, F., Gual, A., Lligoña, A. y Colom, J. (2013). Gender differences in the long-term outcome of alcohol dependence treatments: An analysis of twenty-year prospective follow up. Drug and Alcohol Review, 32, 381-388. doi:10.1111/dar.12023.

Brínez Horta, J. A. (2001). Diferencias de género en problemas con el alcohol, según el nivel de consumo. Adicciones, 13, 439-455. doi:10.20882/adicciones.559.

Caetano, R., Gruenewald, P., Vaeth, P. A. C. y Canino, G. (2018). DSM-5 Alcohol Use Disorder Severity in Puerto Rico: Prevalence, Criteria Profile, and Correlates. Alcoholism, Clinical and Experimental Research, 42, 378-386. doi:10.1111/acer.13572.

Chapman, C., Slade, T., Hunt, C. y Teesson, M. (2015). Delay to first treatment contact for alcohol use disorder. Drug and Alcohol Dependence, 147, 116-121. doi:10.1016/j. drugalcdep.2014.11.029.

Crabb, D. W., Matsumoto, M., Chang, D. y You, M. (2004). Overview of the role of alcohol dehydrogenase and aldehyde dehydrogenase and their variants in the genesis of alcohol-related pathology. The Proceedings of the Nutrition Society, 63, 49-63.

Dawson, D. A., Goldstein, R. B. y Grant, B. F. (2012). Factors associated with first utilization of different types of care for alcohol problems. Journal of Studies on Alcohol and Drugs, 73, 647-656.

Djindjic, N., Jovanovic, J., Djindjic, B., Jovanovic, M. y Jovanovic, J. J. (2012). Associations between the Occupational Stress Index and Hypertension, Type 2 Diabetes Mellitus, and Lipid Disorders in Middle-Aged Men and Women. The Annals of Occupational Hygiene, 56, 10511062. doi:10.1093/annhyg/mes059.

Edelman, E. J. y Fiellin, D. A. (2016). Alcohol Use. Annals of Internal Medicine, 165, 379-380. doi:10.7326/L16-0107.

Edlund, M. J., Booth, B. M. y Han, X. (2012). Who seeks care where? Utilization of mental health and substance use disorder treatment in two national samples of individuals with alcohol use disorders. Journal of Studies on Alcohol and Drugs, 73, 635-646.

European Monitoring Centre for Drugs and Drug Addiction (2015). European Drug Report 2015: Trends and Development. Luxembourg: Publications Office of the European Union.

Grant, B. F., Chou, S. P., Saha, T. D., Pickering, R. P., Kerridge, B. T., Ruan, W. J., ... Hasin, D. S. (2017). Prevalence of 12-Month Alcohol Use, High-Risk Drinking, and DSM-IV Alcohol Use Disorder in the United States, 2001-2002 to 2012-2013. JAMA Psychiatry, 74, 911-923. doi:10.1001/jamapsychiatry.2017.2161.

Hallman, T., Burell, G., Setterlind, S., Odén, A. y Lisspers, J. (2001). Psychosocial risk factors for coronary heart disease, their importance compared with other risk factors and gender differences in sensitivity. Journal of Cardiovascular Risk, 8, 39-49.
Hasin, D. S., O’Brien, C. P., Auriacombe, M., Borges, G., Bucholz, K., Budney, A., ... Grant, B. F. (2013). DSM-5 criteria for substance use disorders: recommendations and rationale. The American Journal of Psychiatry, 170, 834-851. doi:10.1176/appi.ajp.2013.12060782.

Helzer, J. E., van den Brink, W. y Guth, S. E. (2006). Should there be both categorical and dimensional criteria for the substance use disorders in DSM-V? Addiction, 101, 17-22. doi:10.1111/j.1360-0443.2006.01587.x.

Johnson, P. B., Richter, L., Kleber, H. D., McLellan, A. T. y Carise, D. (2005). Telescoping of Drinking-Related Behaviors: Gender, Racial/Ethnic, and Age Comparisons. Substance Use E Misuse, 40, 1139-1151. doi:10.1081/JA200042281.

Kelly, S. J., Ostrowski, N. L. y Wilson, M. A. (1999). Gender differences in brain and behavior: hormonal and neural bases. Pharmacology, Biochemistry, and Behavior, 64, 655664.

Lane, S. P. y Sher, K. J. (2015). Limits of Current Approaches to Diagnosis Severity Based on Criterion Counts. Clinical Psychological Science, 3, 819-835. doi: $10.1177 / 2167702614553026$.

Lieber, C. S. (2000). Ethnic and gender differences in ethanol metabolism. Alcoholism, Clinical and Experimental Research, 24, 417-418.

Mezey, E. (2000). Influence of sex hormones on alcohol metabolism. Alcoholism, Clinical and Experimental Research, 24, 421.

Mirijello, A., D’Angelo, C., Ferrulli, A., Vassallo, G., Antonelli, M., Caputo, F., ... Addolorato, G. (2015). Identification and Management of Alcohol Withdrawal Syndrome. Drugs, 75, 353-365. doi:10.1007/s40265-015-0358-1.

Muthén, B. (2006). Should substance use disorders be considered as categorical or dimensional? Addiction, 101, 6-16. doi:10.1111/j.1360-0443.2006.01583.x.

Nolen-Hoeksema, S. y Hilt, L. (2006). Possible Contributors to the Gender Differences in Alcohol Use and Problems. The Journal of General Psychology, 133, 357-374. doi:10.3200/GENP.133.4.357-374.

Observatorio Español de las Drogas y las Adicciones. (2017). Informe 2017. Alcohol, tabaco y drogas ilegales en España. Consumo problemático de drogas en España, 20062015. Madrid: Ministerio de Sanidad, Política Social e Igualdad.

Observatorio Español de las Drogas y las Toxicomanías. (2011). Informe 2011. Situación y tendencias de los problemas de drogas en España. Madrid: Ministerio de Sanidad, Política Social e Igualdad.

Rehm, J., Manthey, J., Struzzo, P., Gual, A. y Wojnar, M. (2015). Who receives treatment for alcohol use disorders in the European Union? A cross-sectional representative study in primary and specialized health care. European Psychiatry, 30, 885-893. doi:10.1016/j.eurpsy.2015.07.012. 
Saitz, R. (2005). Clinical practice. Unhealthy alcohol use. The New England Journal of Medicine, 352, 596-607. doi:10.1056/NEJMcp042262.

Salvatore, J. E., Cho, S. Bin, y Dick D. M. (2017). Genes, Environments, and Sex Differences in Alcohol Research. Journal of Studies on Alcohol and Drugs, 78, 494-501.

Sanvisens, A., Zuluaga, P., Rivas, I., Rubio, G., Gual, A., Torrens, M., ... Muga, R. (2018). Patients with alcohol use disorder: initial results from a prospective multicenter registry in the Spanish Network on Addiction Disorders. CohRTA Study. Adicciones, 30, 292-300. doi: 10.20882/ adicciones.931

Schwartz, J. y Davaran, A. (2013). Enforcement following $0.08 \%$ BAC law change: Sex-specific consequences of changing arrest practices? Addictive Behaviors, 38, 25062512. doi:10.1016/J.ADDBEH.2013.04.004.

Slade, T., Chiu, W. T., Glantz, M., Kessler, R. C., Lago, L., Sampson, N., ... Degenhardt, L. (2016). A Cross-National Examination of Differences in Classification of Lifetime Alcohol Use Disorder Between DSM-IV and DSM-5: Findings from the World Mental Health Survey. Alcoholism: Clinical and Experimental Research, 40, 17281736. doi:10.1111/acer.13134.

Teesson, M., Hall, W., Slade, T., Mills, K., Grove, R., Mewton, L., ... Haber, P. (2010). Prevalence and correlates of DSM-IV alcohol abuse and dependence in Australia: findings of the 2007 National Survey of Mental Health and Wellbeing. Addiction, 105, 2085-2094. doi:10.1111/ j.1360-0443.2010.03096.x.

White, A., Castle, I. J. P., Chen, C. M., Shirley, M., Roach, D. y Hingson, R. (2015). Converging Patterns of Alcohol Use and Related Outcomes Among Females and Males in the United States, 2002 to 2012. Alcoholism, Clinical and Experimental Research, 39, 1712-1726. doi:10.1111/ acer. 12815 .

White, A. M., Hingson, R. W., Pan, I.J. y Yi, H.Y. (2011). Hospitalizations for alcohol and drug overdoses in young adults ages 18-24 in the United States, 1999-2008: results from the Nationwide Inpatient Sample. Journal of Studies on Alcohol and Drugs, 72, 774-786. doi:10.15288/ JSAD.2011.72.774.

White, A. M., Slater, M. E., Ng, G., Hingson, R. y Breslow, R. (2018). Trends in Alcohol-Related Emergency Department Visits in the United States: Results from the Nationwide Emergency Department Sample, 2006 to 2014. Alcoholism, Clinical and Experimental Research, 42, 352-359. doi:10.1111/acer.13559.

Wodarz, N., Bobbe, G., Eichhammer, P., Weijers, H. G., Wiesbeck, G. A. y Johann, M. (2003). The candidate gene approach in alcoholism: are there gender-specific differences? Archives of Women's Mental Health, 6, 225230. doi:10.1007/s00737-003-0011-y. 